\title{
On the origin of supergravity boundary terms in the AdS/CFT correspondence
}

\author{
G.E.Arutyunov* and S.A.Frolov ${ }^{\dagger}$ \\ Steklov Mathematical Institute, \\ Gubkin str.8, GSP-1, 117966, Moscow, Russia;
}

\begin{abstract}
The standard formulation of the AdS/CFT correspondence is incomplete since it requires adding to a supergravity action some a priori unknown boundary terms. We suggest a modification of the correspondence principle based on the Hamiltonian formulation of the supergravity action, which does not require any boundary terms. Then all the boundary terms of the standard formulation naturally appear by passing from the Hamiltonian version to the Lagrangian one. As examples the graviton part of the supergravity action on the product of $A d S_{d+1}$ with a compact Einstein manifold $\mathcal{E}$ and fermions on $A d S_{d+1}$ are considered. We also discuss conformal transformations of gravity fields on the boundary of $A d S$ and show that they are induced by the isometries of $A d S$.
\end{abstract}

\section{Introduction}

According to a recent conjecture by Maldacena [1] the large $N$ limit of certain conformal field theories in $d$ dimensions can be governed by supergravity on the product of $d+1$-dimensional anti de Sitter space $A d S_{d+1}$ with a compact manifold $\mathcal{E}$. This conjecture was further elaborated by Gubser, Klebanov and Polyakov [2] and Witten [3], who proposed to identify the generating functional of the connected Green functions in a $d$-dimensional conformal field theory with the minimum of the supergravity action subject to certain conditions imposed on supergravity fields at the boundary of $A d S_{d+1} \times \mathcal{E}$. In [4]-[12] the proposal was used to compute some twoand three-point correlation functions in $\mathcal{N}=4 S U(N)$ Yang-Mills theory, which exhibit the expected conformal behavior. However, to make the AdS/CFT correspondence complete one has to add to the supergravity action some boundary terms.

The necessity of the boundary terms can be seen as follows. Firstly, to calculate the Green functions in field theory one has to introduce the UV regularization, for example, the lattice regularization with the lattice step $a$. Since any regularization violates the conformal invariance,

\footnotetext{
*arut@genesis.mi.ras.ru

${ }^{\dagger}$ Address after September 1, 1998: The University of Alabama, Department of Physics and Astronomy, Box 870324, Tuscaloosa, Alabama 35487-0324.
} 
one has to add some counterterms to the field theory action, to restore (if possible) the conformal invariance in the limit $a \rightarrow 0$.

Secondly, recall that the geometry of the $A d S_{d+1}$ space is described by the metric

$$
d s^{2}=\frac{1}{x_{0}^{2}}\left(\eta_{i j} d x^{i} d x^{j}+d x_{0}^{2}\right),
$$

where $\eta_{i j}$ is the $d$-dimensional Minkowski metric. The boundary of $A d S_{d+1}$ space is at $x_{0}=0$ and can be identified with the Minkowski space. The boundary is located at infinite distance from any point of $A d S_{d+1}$. This leads to infrared divergency of the gravity action. To make the action finite 1 one should cut $A d S_{d+1}$ space off at $x_{0}=\varepsilon$ and consider the part of $A d S_{d+1}$ with $x_{0} \geq \varepsilon$. The minimum of the gravity action is calculated by requiring the gravity fields to vanish at $x_{0}=\infty$ and to take arbitrary values at $x_{0}=\varepsilon$.

The IR regularization leads to explicit violation of the conformal invariance of the gravity action and to restore it in the limit $\varepsilon \rightarrow 0$ one has to add some boundary terms. In fact, the gravity action considered as a functional of the boundary fields is never conformal invariant [13]. The conformal invariance of the $A d S_{d+1}$ gravity should be understood as the validity of the conformal Ward identities for correlation functions? It is clear from this discussion that the UV regularization and the counterterms of the field theory correspond to the IR regularization and the boundary terms of the gravity theory, the UV cutoff $a$ being identified with the IR cutoff $\varepsilon$.

Then the standard formulation of the AdS/CFT correspondence [2, 3] asserts that a connected Green function in the $d$-dimensional conformal field theory is equal to

$$
\lim _{a \rightarrow 0}\left\langle\mathcal{O}_{1}\left(x_{1}\right) \ldots \mathcal{O}_{n}\left(x_{n}\right)\right\rangle=\left.\lim _{\varepsilon \rightarrow 0} \frac{\delta}{\delta \phi_{1}\left(x_{1}\right)} \cdots \frac{\delta}{\delta \phi_{n}\left(x_{n}\right)} \min S_{g r}\left(\phi_{1}, \ldots, \phi_{n}\right)\right|_{\phi_{i}\left(x, x_{0}=\varepsilon\right)=\phi_{i}(x)}
$$

where $\mathcal{O}_{i}\left(x_{i}\right)$ are some gauge-invariant operators in the field theory and $\phi_{i}\left(x_{i}\right)$ are the gravity fields corresponding to $\mathcal{O}_{i}\left(x_{i}\right)$. The gravity action $S_{g r}\left(\phi_{1}, \ldots, \phi_{n}\right)$ is the sum of the bulk action and the boundary terms. Note that the boundary terms that depend locally on the boundary fields $\phi_{i}(x)$ (i.e. do not depend on $\left.\partial_{0} \phi_{i}(x)\right)$ do not contribute to the Green functions. From the viewpoint of the boundary CFT these local boundary terms reflect the usual ambiguity in the definition of the $T$-product. Since the conformal invariant correlation functions are not distributions, one can not use the Fourier image of the Green functions in eq.(1.1). Loosely speaking, the generating functional of the Green functions coincides with the partition function of the supergravity (string) theory.

It is worth noting that since the boundary terms are in general unknown, the usual formulation of the AdS/CFT correspondence is incomplete. Nevertheless, it seems possible to formulate the AdS/CFT correspondence in a way that does not depend on any unknown boundary terms. To this end remind that in quantum mechanics the minimum of an effective action with given initial $q_{i n}$ and final $q_{f}$ configurations is proportional to the logarithm of the transition amplitude from the initial state $\mid q_{i n}>$ to the final state $\mid q_{f}>$. Note that the effective action one gets from

\footnotetext{
${ }^{1}$ Strictly speaking, the regularized action is still infinite because of the infinite volume of the boundary. This infinity is cured up by adding proper boundary terms.

${ }^{2}$ Recently an attempt [14] to present a general proof of conformal invariance of the CFT Green functions computed from supergravity on $A d S$ background was made. However, the arguments of [14] do not take into account IR divergencies and rely on inproper transformation laws of boundary fields.
} 
the transition amplitude is always of the form $\int d t(p \dot{q}-H(p, q))$ without any boundary terms since the initial and final states are taken in the coordinate representation. Coming back to the AdS/CFT correspondence we see that the coordinate $x_{0}$ plays a distinguished role. If we introduce a new coordinate $t=\log x_{0}$ and interpret $t$ as the time coordinate in $A d S$, then we can postulate that the generating functional of the Green functions coincides with the string transition amplitude from the initial state $\mid \phi>$ to the "vacuum" state $\mid 0>$ :

$$
Z(\phi)=\lim _{\substack{t^{\prime} \rightarrow-\infty \\ t^{\prime \prime} \rightarrow+\infty}}<0\left|\mathrm{e}^{-i \int_{t^{\prime}}^{t^{\prime \prime}} H_{s t r}(t) d t}\right| \phi>,
$$

where $H_{s t r}$ is the string theory Hamiltonian governing the dynamics in the $t$ direction.

In the classical supergravity limit one recovers the above-mentioned version of the AdS/CFT correspondence with the supergravity boundary terms fixed by the Hamiltonian formulation of the supergravity action. It means that all the boundary terms naturally appear by passing from the Hamiltonian version to the Lagrangian one. In particular, a local boundary term appears in the bulk as a total derivative term. From the Hamiltonian point of view such a term can be removed by performing a canonical transformation affecting only the momenta. However, a choice of momenta is partially fixed by requiring the minimum of the Hamiltonian to be achieved at $p=0=\phi$. Such a choice of momenta would ensure the vanishing of the one-point correlation functions.

Since supergravity is a constrained system, to obtain its Hamiltonian formulation one has to impose some gauge conditions and to eliminate the second-class constraints. The most convenient gauges seem to be a generalization of the temporal gauge $A_{0}=0$ in the Yang-Mills theory. In these gauges the gravity action has a residual symmetry with gauge parameters that do not depend on $t$. The residual symmetry leads to conservation laws for currents of the boundary field theory. Moreover, on the boundary of $A d S$ the residual gauge group gets enhanced and includes, in particular, isometries of the background space. In the paper we show that conformal transformations of the supergravity boundary fields are induced by the isometries.

In this paper we demonstrate how the Hamiltonian version of the AdS/CFT correspondence permits to determine the supergravity boundary terms that have to be added to the standard supergravity action.

The plan of the paper is as follows. In the second Section we consider a gravity model on the $A d S \times \mathcal{E}$ background, where $\mathcal{E}$ is an arbitrary Einstein manifold. We show that the Hamiltonian formulation correctly predicts the standard boundary term [15] as well as the term proportional to the volume of the boundary suggested in 8 ] for the case of the gauged supergravity on $A d S_{d+1}$ describing the dynamics of zero modes in $A d S_{d+1} \times \mathcal{E}$ compactification. There is also an additional term which, however, is a local functional of boundary fields and, thereby, does not change the value of the Green functions. The boundary terms discussed in the second Section can be equivalently found relying on the conformal invariance of the corresponding boundary field theory. Namely, the conformal invariance requires any one-point correlation function to vanish, and, therefore, the minimum of the supergravity action extended over the $A d S_{d+1} \times \mathcal{E}$ background should not have linear dependence on the boundary values of the supergravity fields. In this Section we also consider the conformal transformations of the boundary graviton induced by the isometries of $A d S$. 
In the third Section we discuss fermions on the $A d S$ background and demonstrate that the fermion boundary term suggested in [5] again follows from the Hamiltonian version of the action. We also study conformal transformations of the boundary fermion fields.

\section{Metric dependent boundary terms}

Clearly, the boundary terms depending only on the metric originate from the Einstein-Hilbert term

$$
I=\int_{M} d^{D} x \sqrt{-g} R
$$

where we choose the gravitational constant equal to one. We consider the theory on a manifold with a boundary $\partial M$. By using the reparametrization invariance of action (2.2) we can describe $\partial M$ at least locally as a hypersurface $t=\log \varepsilon=$ const and choose in the vicinity of $\partial M$ a gauge $g_{t t}=1$ and $g_{t \mu}=0$.

Remind that to study the Hamiltonian formulation it is useful to represent $\sqrt{-g} R$ in the following form

$$
\sqrt{-g} R=\sqrt{-g} g^{a b}\left(\Gamma_{a d}^{c} \Gamma_{c b}^{d}-\Gamma_{a b}^{c} \Gamma_{c d}^{d}\right)+\partial_{a}\left(\sqrt{-g} g^{b c} \Gamma_{b c}^{a}-\sqrt{-g} g^{a b} \Gamma_{b c}^{c}\right) .
$$

The last term is a total derivative term and should be omitted in the Hamiltonian formulation.

Considering a manifold with a boundary one usually adds to (2.2) the standard boundary term that removes all terms linear in second derivatives [15]:

$$
I^{(1)}=2 \int_{\partial M} d^{D-1} x \sqrt{-\bar{g}} K .
$$

Here $K$ is a trace of the second fundamental form on the boundary and $\bar{g}$ is the determinant of the induced metric. Remind that introducing the normal $n^{a}$ to the boundary, $K$ can be written as $K=\nabla_{a} n^{a}$. In our gauge the induced metric coincides with $g_{\mu \nu}, g=\bar{g}$ and $n^{a}=(-1,0, \ldots, 0)$. Therefore,

$$
I^{(1)}=2 \int_{\partial M} d^{D-1} x \sqrt{-\bar{g}} \frac{1}{\sqrt{-g}} \partial_{a}\left(\sqrt{-g} n^{a}\right)=-2 \int_{\partial M} d^{D-1} x \partial_{t}(\sqrt{-\bar{g}}) .
$$

Now one can easily see that integrating the total derivative term in (2.3) one obtains $-I^{(1)}$. Thus, adding to (2.2) boundary term $I^{(1)}$ we get the action that should be used to obtain the Hamiltonian formulation. It is worth noting that $I^{(1)}$ is not cancelled by the total derivative term in (2.2) in an arbitrary gauge. However, the difference is always a local functional of boundary fields and, therefore, is irrelevant for computing the Green functions.

Thus, the action $S=I+I^{(1)}$ acquires a form

$$
S=\int_{M} d^{D} x \sqrt{-g}\left(\frac{1}{4} \partial_{t} g^{\mu \nu} \partial_{t} g_{\mu \nu}+\frac{1}{4} g^{\mu \nu} \partial_{t} g_{\mu \nu} g^{\rho \lambda} \partial_{t} g_{\rho \lambda}+g^{\mu \nu}\left(\Gamma_{\mu \rho}^{\lambda} \Gamma_{\lambda \nu}^{\rho}-\Gamma_{\mu \nu}^{\lambda} \Gamma_{\lambda \rho}^{\rho}\right)\right) .
$$

Suppose that a vacuum solution of the Einstein equations defines the following metric

$$
d s^{2}=d t^{2}+G_{\mu \nu} d x^{\mu} d x^{\nu}=d t^{2}+e^{-2 t} \eta_{i j} d x^{i} d x^{j}+G_{\alpha \beta} d x^{\alpha} d x^{\beta}
$$


on the manifold $M=A d S_{d+1} \times \mathcal{E}_{p}$, where $\mathcal{E}_{p}$ is a compact Einstein manifold with the metric $G_{\alpha \beta}: R_{\alpha \beta}=\nu G_{\alpha \beta}$. Here $i, j=1, \ldots, d ; \alpha, \beta=1, \ldots, p, D=d+1+p$ and $\eta_{i j}$ is the metric of the $d$-dimensional Minkowski space. The boundary of $M$ is a hypersurface $t=\log \varepsilon=$ const, which can be considered as the product of the Minkowski space with $\mathcal{E}_{p}$.

It is convenient to decompose the metric as follows $g_{\mu \nu}=G_{\mu \rho} H_{\nu}^{\rho}$. Then, (2.6) reads as

$$
\begin{aligned}
S & =\int_{M} d^{D} x \sqrt{-G} \sqrt{H}\left(-d(d-1)+\frac{1}{4} \partial_{t} H_{\mu}^{\nu} \partial_{t}\left(H^{-1}\right)_{\nu}^{\mu}+\frac{1}{4}\left(\partial_{t} H_{\mu}^{\nu}\left(H^{-1}\right)_{\nu}^{\mu}\right)^{2}\right. \\
& \left.-\partial_{t} H_{\mu}^{\alpha}\left(H^{-1}\right)_{a}^{\mu}+g^{\mu \nu}\left(\Gamma_{\mu \rho}^{\lambda} \Gamma_{\lambda \nu}^{\rho}-\Gamma_{\mu \nu}^{\lambda} \Gamma_{\lambda \rho}^{\rho}\right)\right)+2(1-d) \int_{M} d^{D} x \partial_{t} \sqrt{-g}
\end{aligned}
$$

The last term is a total derivative term and should be omitted in passing to the Hamiltonian version. From the Lagrangian point of view this total derivative term can be cancelled by adding to (2.8) the boundary term proportional to the volume of the boundary:

$$
I^{(2)}=2(1-d) \int_{\partial M} \sqrt{-\bar{g}}
$$

As was mentioned in the Introduction one should require the minimum of the Hamiltonian to be achieved at zero value of coordinates and momenta. The only term that could shift the value of momenta is $-\sqrt{-g} \partial_{t} H_{\mu}^{\alpha}\left(H^{-1}\right)_{a}^{\mu}$. Indeed, expanding this term near the background one gets

$$
-\partial_{t}\left(\sqrt{-G} h_{\alpha}^{\alpha}\right)-d \sqrt{-G} h_{\alpha}^{\alpha}+O\left(h^{2}\right),
$$

where $H_{\mu}^{\nu}=\delta_{\mu}^{\nu}+h_{\mu}^{\nu}$. The first term in this expansion is a total derivative term linear in $h$ and, therefore, shifts the value of momenta. It turns out that from the Lagrangian point of view there exists a lot of covariant boundary terms that can compensate this shift. For example, one can add to action (2.8) the following term

$$
\int_{\partial M} \sqrt{-\bar{g}} f(\bar{R})
$$

where $\bar{R}$ is the curvature of the metric induced on the boundary. The function $f(x)$ should satisfy two conditions: $f(\nu p)=0$ and $f^{\prime}(\nu p)=1 / \nu$. A possible choice is $f(x)=x-\frac{1}{\nu p} x^{2}$. Thus, the action that admits a straightforward Hamiltonization and should be used in computation of the Green functions has the form

$$
\mathbf{S}=\int_{M} d^{D} x \sqrt{-g} R+2 \partial_{n} \int_{\partial M} d^{D-1} x \sqrt{\bar{g}}+2(1-d) \int_{\partial M} d^{D-1} x \sqrt{\bar{g}}+\int_{\partial M} d^{D-1} x \sqrt{\bar{g}} f(\bar{R}) .
$$

To compare the boundary terms (2.11) with the ones introduced for the pure $A d S_{d+1}$ gravity [8] one obviously has to perform the dimensional reduction of $\mathbf{S}$ to $D=d+1$ dimensions. Leaving only the graviton modes one gets

$\mathbf{S}=\int_{M} d^{d+1} x \sqrt{-g}(R+d(d-1))+2 \partial_{n} \int_{\partial M} d^{d} x \sqrt{\bar{g}}+2(1-d) \int_{\partial M} d^{d} x \sqrt{\bar{g}}+\int_{\partial M} d^{d} x \sqrt{\bar{g}} f(\bar{R})$,

where an overall multiplier being the volume of the sphere is omitted. Here $d(d-1)$ is the cosmological constant needed to ensure the consistency of the reduction. The first two boundary 
terms are exactly the same as in [8], where they were added to cancel boundary terms that are linear in metric perturbation of the action of $D=d+1$ dimensional gravity near $A d S_{d+1}$ background. Since $f(0)=0$ after the reduction and $\bar{R}=0$ on the background the third boundary term does not contribute to the first variation of (2.12). The boundary area counterterm is needed just for cancelling the linear terms ensuring thereby the vanishing of onepoint Green functions. Only the second fundamental form is important for computing the higher-point Green functions. The other boundary terms are local and as was discussed in the Introduction do not contribute.

Now we discuss the conformal transformations of the graviton on the boundary induced by isometries of $A d S$. In the sequel it is convenient to deal with the coordinate $x_{0}=\mathrm{e}^{t}$ and to decompose $g_{a b}=G_{a b}+h_{a b}$. Denote by $\xi^{a}$ a Killing vector of the background metric. Assuming $G$ to be stationary under diffeomorphism generated by $\xi$ one finds that transformation

$$
\delta h_{a b}=\xi^{c} \partial_{c} h_{a b}+h_{a c} \partial_{b} \xi^{c}+h_{b c} \partial_{a} \xi^{c}
$$

is a rigid symmetry of equations of motion in every order in metric perturbation $h_{a b}$. Note that the Killing vectors of the $A d S_{d+1}$ background can be written as

$$
\begin{aligned}
& \xi^{0}=x_{0}\left(A_{k} x^{k}+D\right), \\
& \xi^{i}=-\frac{x_{0}^{2}-\varepsilon^{2}}{2} A^{i}+\left(-\frac{1}{2}\left(A^{i} x^{2}-2 x^{i} A_{k} x^{k}\right)+D x^{i}+\Lambda_{j}^{i} x^{j}+P^{i}\right),
\end{aligned}
$$

where $A^{i}, D, \Lambda_{j}^{i}, P^{i}$ generate on the boundary special conformal transformations, dilatations, Lorentz transformations and shifts respectively. Transformations (2.13) do not respect the gauge conditions $h_{0 i}=0: \delta h_{0 i}=h_{i j} \partial_{0} \xi^{j}$. However, the gauge can be restored by combining (2.14) with the transformation $\delta h_{a b}=\nabla_{a} \chi_{b}+\nabla_{b} \chi_{a}$ with $\chi^{0}=0$ and $\chi^{i}=\int_{\varepsilon}^{x_{0}} z g^{k j} h_{i j}(z, \vec{x}) A_{k}$. Now it is easy to find

$$
\delta h_{i}^{j}=\xi^{k} \partial_{k} h_{i}^{j}+h_{i}^{k} \partial^{j} \xi_{k}+h_{k}^{j} \partial_{i} \xi^{k}+\xi^{0} \partial_{0} h_{i}^{j}-\frac{2}{x_{0}} \xi^{0} h_{i}^{j}+\partial_{i} \chi^{j}+\partial^{i} \chi_{j},
$$

where $h_{i}^{j}=G^{j k} h_{i k}$.

Note that on the boundary $\chi^{i}$ vanish. Clearly, to obtain the induced transformations on the boundary one should know the behavior of $\partial_{0} h_{i}^{j}$ in the limit $\varepsilon \rightarrow 0$. To this end we consider the linearized equations of motion following from (2.12):

$$
\nabla_{k} \nabla^{k} h_{a b}+\nabla_{a} \nabla_{b} h-\nabla_{a} \nabla_{c} h_{b}^{c}-\nabla_{b} \nabla_{c} h_{a}^{c}+2\left(h_{a b}-g_{a b} h\right)=0 .
$$

Eq.(2.16) has the standard symmetry $\delta h_{a b}=\nabla_{a} \xi_{b}+\nabla_{b} \xi_{a}$ that allows one to impose the gauge conditions $h_{0 a}=0$. Below, to simplify the notation, we adopt the convention that the indices $i, j$ are raised and lowered by using the Minkowski metric $\eta_{i j}$, in particular, $\square=\eta^{i j} \partial_{i} \partial_{j}$. In this gauge covariant equation (2.16) written for $h_{i}^{j}$ acquires the form

$$
\partial_{0}^{2} h_{i}^{j}+\square h_{i}^{j}+\frac{1-d}{x_{0}} \partial_{0} h_{i}^{j}-\frac{1}{x_{0}} \delta_{i}^{j} \partial_{0} h+\left(\partial_{i} \partial^{j} h-\partial_{i} \partial^{k} h_{k}^{j}-\partial^{j} \partial_{k} h_{k}^{i}\right)=0 .
$$

In addition one has two constraints:

$$
\begin{aligned}
& \partial_{0}\left(\partial_{i} h-\partial_{k} h_{i}^{k}\right)=0, \\
& \square h+\frac{(1-d)}{x_{0}} \partial_{0} h-\partial_{i} \partial^{j} h_{j}^{i}=0,
\end{aligned}
$$


which follow from the equation for $h_{0 a}$. Introduce the transversal part $h_{i}^{\perp j}$ of $h_{i}^{j}$ :

$$
h_{i}^{\perp j} \equiv h_{i}^{j}-\frac{1}{\square} \partial_{i} \partial^{k} h_{k}^{j}-\frac{1}{\square} \partial^{j} \partial_{k} h_{i}^{k}+\frac{\partial_{i} \partial^{j}}{\square^{2}} \partial_{k} \partial^{m} h_{m}^{k},
$$

The trace $h^{\perp}$ of the transversal part satisfies $\square\left(h-h^{\perp}\right)=\partial_{i} \partial^{j} h_{j}^{i}$.

Obviously, by using (2.18) one gets

$$
\partial_{0} h=\partial_{0} h^{\perp}+\frac{1}{\square} \partial^{i} \partial_{0} \partial_{j} h_{i}^{j}=\partial_{0} h^{\perp}+\partial_{0} h .
$$

Thus,

$$
\partial_{0} h^{\perp}=0, \quad \partial_{0} h=\frac{x_{0}}{d-1} \square h^{\perp},
$$

where the last equation follows immediately from eq.(2.19).

Introduce a traceless transversal part $\bar{h}_{i}^{j}$ of $h_{i}^{j}$ :

$$
\bar{h}_{i}^{j}=h_{i}^{\perp j}+\frac{1}{d-1}\left(\frac{\partial_{i} \partial^{j}}{\square}-\delta_{i}^{j}\right) h^{\perp} .
$$

Then with the account of (2.21) eq.(2.17) can be written as the following equation on $\bar{h}_{i}^{j}$ :

$$
\partial_{0}^{2} \bar{h}_{i}^{j}+\square \bar{h}_{i}^{j}+\frac{1-d}{x_{0}} \partial_{0} \bar{h}_{i}^{j}=0,
$$

which coincides with the equation for a free massless scalar field on the AdS background. The Fourier mode solution $\bar{h}_{i}^{j}\left(x_{0}, \vec{k}\right)$ of eq. (2.23) obeying the boundary condition $\bar{h}_{i}^{j}(\varepsilon, \vec{k})=h_{i}^{j}(\vec{k})$, where $h_{i}^{j}(\vec{k})$ is an arbitrary transversal tensor, reads as

$$
\bar{h}_{i}^{j}\left(x_{0}, \vec{k}\right)=\left(\frac{x_{0}}{\varepsilon}\right)^{d / 2} \frac{K_{d / 2}\left(x_{0} k\right)}{K_{d / 2}(\varepsilon k)} h_{i}^{j}(\vec{k}),
$$

where $K_{d / 2}$ is the Mackdonald function and $k=|\vec{k}|$. It is clear from (2.24) that $\partial_{0} \bar{h}_{i}^{j}(\varepsilon, \vec{k})=$ $O(\varepsilon)$. By using eqs.(2.18) and (2.19) one can see that $\partial_{0} \partial_{j} h_{i}^{j}(\varepsilon, \vec{k})=O(\varepsilon)$ and, therefore, $\partial_{0} h_{i}^{j}(\varepsilon, \vec{k})=O(\varepsilon)$.

Thus, coming back to eq.(2.15), in the limit $\varepsilon \rightarrow 0$ we are left with the following transformation law induced on the boundary

$$
\delta h_{i}^{j}(\vec{x})=\xi^{k} \partial_{k} h_{i}^{j}+h_{i}^{k} \partial^{j} \xi_{k}+h_{k}^{j} \partial_{i} \xi^{k}-2\left(A_{k} x^{k}+D\right) h_{i}^{j},
$$

where $\xi^{i}=\xi^{i}(\varepsilon, \vec{x})$. This transformation law shows, in particular, that under dilatations the boundary graviton transforms with scaling dimension zero as required by the AdS/CFT correspondence since it couples with the stress-energy tensor whose scaling dimension is $d$.

As a byproduct of our consideration we can easily derive the two-point Green function for the stress-energy tensor in the boundary CFT. Quadratic part of action (2.12) computed on the solution of equations of motion reduces to the following form

$$
\mathbf{S}^{(I I)}=\frac{1}{4} \int d^{d} x \varepsilon^{1-d}\left(h_{i}^{j} \partial_{0} h_{j}^{i}-h \partial_{0} h\right),
$$


where we have omitted all irrelevant local terms. Using eqs.(2.21) it is easy to show that

$$
h_{i}^{j} \partial_{0} h_{j}^{i}-h \partial_{0} h=\bar{h}_{i}^{j} \partial_{0} \bar{h}_{j}^{i}-\frac{x_{0}}{d-1} h^{\perp} \square h^{\perp},
$$

and, therefore,

$$
\mathbf{S}^{(I I)}=\frac{1}{4} \int d^{d} x \varepsilon^{1-d} \bar{h}_{i}^{j} \partial_{0} \bar{h}_{j}^{i}
$$

where again the local term was omitted. Since $\bar{h}_{i}^{j}$ satisfies the massless scalar field equation on the $A d S$ background eq.(2.27) leads to the expected two-point Green function of the stressenergy tensor. Note that our computation of the Green function essentially differs from the one in [8]. In particular, we do not impose the requirement of vanishing the trace of the graviton. In [8] $h$ was put equal to zero by arguing that otherwise it blows up at infinity. However, eq.(2.21) is the first order equation and, therefore, one can not require any condition at infinity. Moreover, the AdS/CFT correspondence implies the decoupling of $h$ on the boundary since, otherwise, the trace of the stress-energy tensor would not be equal to zero. The fact of decoupling of $h$ should be checked explicitly. In our computation scheme the contribution of $h$ results only in a local boundary term. In principle, this contribution can be cancelled by choosing in (2.12) a proper function $f$.

Another representative example demonstrating the relation between the transformation law in the bulk and the conformal properties of the boundary data is provided by a massive scalar $\phi$ on $A d S_{d+1}$ background. The equation of motion for $\phi$

$$
\partial_{0}^{2} \phi+\frac{1-d}{x_{0}} \partial_{0} \phi+\left(\square-\frac{m^{2}}{x_{0}^{2}}\right) \phi=0
$$

has a Fourier mode solution

$$
\phi\left(x_{0}, \vec{k}\right)=\left(\frac{x_{0}}{\varepsilon}\right)^{\frac{d}{2}} \frac{K_{\nu}\left(x_{0} k\right)}{K_{\nu}(\varepsilon k)} \phi_{0}(\vec{k}), \quad \nu^{2}=m^{2}+\frac{d^{2}}{4}
$$

that equals to $\phi_{0}(\vec{k})$ on the boundary. Under the $A d S$ isometries (2.14) the scalar $\phi$ transforms as

$$
\delta \phi\left(x_{0}, \vec{x}\right)=\xi^{a} \partial_{a} \phi=\xi^{i} \partial_{i} \phi+\xi^{0} \partial_{0} \phi
$$

and, therefore, the transformation properties of the boundary value $\phi_{0}$ are determined by $\lim _{x_{0} \rightarrow \varepsilon} \xi^{0} \partial_{0} \phi$. Computing the derivative of $\phi$ in the bulk direction one finds $\partial_{0} \phi(\varepsilon, \vec{x})=(d / 2-$ $\nu) \varepsilon^{-1} \phi_{0}(\vec{x})+O(1)$ and, therefore,

$$
\delta \phi_{0}(\vec{x})=\xi^{i}(\vec{x}) \partial_{i} \phi_{0}(\vec{x})+\left(\frac{d}{2}-\nu\right)\left(A_{k} x^{k}+D\right) \phi_{0}(\vec{x}),
$$

where $\xi^{i}(\vec{x})=\xi^{i}(\varepsilon, \vec{x})$ Thus, at the boundary $\phi$ couples with the operator of conformal dimension $\Delta=d / 2+\nu$ as it should be.

These examples show that the conformal properties of boundary data are not completely determined by the transformation law of the corresponding bulk fields but depend essentially on the explicit form of the interaction in the bulk. One could imagine such an exotic interaction that would lead to a nonlinear or nonlocal realization of the conformal group on the boundary. 


\section{$3 \quad$ Fermions on the $A d S$ background}

We start with the action for the free massive Dirac fermion on the $A d S$ background

$$
S_{\psi}(\alpha)=\int_{M} d^{d+1} x \sqrt{-G}\left(\frac{\alpha}{2} \bar{\psi} \Gamma^{\mu} D_{\mu} \psi-\frac{\beta}{2} D_{\mu} \bar{\psi} \Gamma^{\mu} \psi-m \bar{\psi} \psi\right),
$$

where $\alpha+\beta=2$. Note that actions with different $\alpha$ and $\beta$ differ only by a total derivative. In the sequel we denote by $\mu, \nu, \ldots$ and by $a, b, .$. the world and the tangent indices respectively, $\Gamma^{\mu}=e_{a}^{\mu} \gamma^{a}$ and $\gamma_{a}$ are the Dirac matrices of $d+1$ dimensional Minkowski space: $\gamma_{a} \gamma_{b}+\gamma_{b} \gamma_{a}=$ $2 \eta_{a b}$. Here the conjugated fermion is defined as $\bar{\psi}=\psi^{*} \gamma^{1}$ since $x^{1}$ is the time direction of the boundary field theory.

Introduce the left and right chiral components of $\psi: \psi_{ \pm}=\frac{1}{2}\left(1 \pm \gamma_{0}\right) \psi$ and define $\bar{\psi}_{ \pm}=$ $\left(\psi_{ \pm}\right)^{*} \gamma^{1}$. Note that $\bar{\psi}_{ \pm} \gamma^{0}=\mp \bar{\psi}_{ \pm}$.

Action (3.32) is already written in the first-order formalism. Therefore, either $\psi_{-}$or $\psi_{+}$plays the role of the coordinate. In order to understand what component of $\psi$ should be regarded as the coordinate we discuss the transformation properties of $\psi$ at the boundary under isometries of the $A d S$ background.

Recall that under the coordinate transformation generated by $\xi^{\mu}$ the veilbein $e_{\mu}^{a}$ transforms as follows

$$
\delta e_{\mu}^{a}=\xi^{\rho} \nabla_{\rho} e_{\mu}^{a}+\nabla_{\mu} \xi^{\rho} e_{\rho}^{a}
$$

where $\nabla_{\rho}$ is a covariant derivative with respect to the background metric $G_{\mu \nu}$. If $\xi^{\mu}$ is a Killing vector of $G_{\mu \nu}$, then $\nabla_{\mu} \xi_{\lambda}=\nabla_{[\mu} \xi_{\lambda]}$. In addition to (3.33) one also has the local Lorentz symmetry $\delta e_{\mu}^{a}=\Lambda_{b}^{a}(x) e_{\mu}^{b}$. Thus, the general symmetry transformation is

$$
\delta e_{\mu}^{a}=\xi^{\rho} \nabla_{\rho} e_{\mu}^{a}+\nabla_{\mu} \xi^{\rho} e_{\rho}^{a}+\Lambda_{b}^{a} e_{\mu}^{b}
$$

However, in a fixed background we are left with the transformations that preserve $e_{\mu}^{a}: \delta e_{\mu}^{a}=0$. This condition can be attained by choosing the following matrix $\Lambda$ :

$$
\Lambda^{a b}=\xi^{\rho} \omega_{\rho}^{a b}+\nabla_{[\mu} \xi_{\nu]} e^{a \mu} e^{b \nu}
$$

where $\omega_{\rho}^{a b}$ is a spin connection. For the $A d S$ background we choose $e_{0}^{0}=\frac{1}{x_{0}}, e_{0}^{i}=e_{i}^{0}=0$ and $e_{i}^{j}=\frac{1}{x_{0}} \delta_{i}^{j}$ and, therefore, $\omega_{\mu}^{i j}=0, \omega_{\mu}^{0 i}=\frac{1}{x_{0}} \delta_{\mu}^{i}$. Computing (3.35), one finds $\Lambda^{0 i}=\partial_{0} \xi^{i}$ and $\Lambda^{i j}=\eta^{k[i} \partial_{k} \xi^{j]}$.

A spinor $\psi$ on the $A d S$ background transforms under isometries just as a scalar. However, if we combine isometries with the local Lorentz transformations generated by $\Lambda^{a b}$ (3.35), the transformation law for $\psi$ modifies as follows

$$
\delta \psi=\xi^{\mu} \partial_{\mu} \psi-\frac{1}{4} \Lambda^{a b} \gamma_{a b} \psi
$$

With the account of the explicit form of $\Lambda^{a b}$ the last formula acquires the form

$$
\delta \psi=\xi^{i} \partial_{i} \psi+\xi^{0} \partial_{0} \psi-\frac{1}{2} \partial_{0} \xi^{i} \gamma_{0 i} \psi-\frac{1}{4} \eta^{k[i} \partial_{k} \xi^{j]} \gamma_{i j} \psi
$$


Projecting $(3.36)$ on $\psi_{\mp}$, one gets

$$
\delta \psi_{\mp}=\xi^{i} \partial_{i} \psi_{\mp}+\xi^{0} \partial_{0} \psi_{\mp} \pm \frac{1}{2} \partial_{0} \xi^{i} \gamma_{i} \psi_{ \pm}-\frac{1}{4} \eta^{k[i} \partial_{k} \xi^{j]} \gamma_{i j} \psi_{\mp} .
$$

As was shown in 15, 10] a solution of the Dirac equation vanishing at infinity has the form

$$
\psi_{ \pm}\left(x_{0}, \vec{k}\right)=\left(\frac{x_{0}}{\varepsilon}\right)^{\frac{d+1}{2}} \frac{K_{m \mp \frac{1}{2}}\left(x_{0} k\right)}{K_{m \mp \frac{1}{2}}(\varepsilon k)} \psi_{ \pm}(\vec{k}) .
$$

In addition one has the following relation between $\psi_{+}$and $\psi_{-}$:

$$
\psi_{+}\left(x_{0}, \vec{k}\right)=-i \frac{\gamma^{i} k_{i}}{k} \frac{K_{m-\frac{1}{2}}\left(k x_{0}\right)}{K_{m+\frac{1}{2}}\left(k x_{0}\right)} \psi_{-}\left(x_{0}, \vec{k}\right) .
$$

For the sake of clarity we restrict the subsequent consideration to the case $m>0$. Then in the limit $\varepsilon \rightarrow 0$ from (3.38) one has $\psi_{+}(\vec{x})=\varepsilon \gamma^{i} \partial_{i} \psi_{-}(\vec{x})+O(\varepsilon)$, while (3.37) allows one to determine the boundary value of $\partial_{0} \psi_{ \pm}$:

$$
\partial_{0} \psi_{ \pm}(\varepsilon, \vec{x})=\frac{1}{\varepsilon}\left(\frac{d+1}{2}-m \pm \frac{1}{2}\right) \psi_{ \pm}(\vec{x})+O(1)
$$

Thus, the transformations of $\psi_{ \pm}$induced on boundary acquire the form

$$
\begin{aligned}
& \delta \psi_{-}(\varepsilon, \vec{x})=\xi^{i} \partial_{i} \psi_{-}+w\left(A_{k} x^{k}+D\right) \psi_{-}-\frac{\varepsilon^{2}}{2} \gamma^{i} \gamma^{j} A_{i} \partial_{j} \psi_{-}-\frac{1}{4} \eta^{k[i} \partial_{k} \xi^{j]} \gamma_{i j} \psi_{-}+O(\varepsilon), \\
& \delta \psi_{+}(\varepsilon, \vec{x})=\xi^{i} \partial_{i} \psi_{+}+(w+1)\left(A_{k} x^{k}+D\right) \psi_{+}-\frac{1}{2} \frac{1}{\square} \gamma^{i} \gamma^{j} A_{i} \partial_{j} \psi_{+}-\frac{1}{4} \eta^{k[i} \partial_{k} \xi^{j]} \gamma_{i j} \psi_{+}+O(\varepsilon),
\end{aligned}
$$

where $w=\frac{d}{2}-m$. Therefore, on the boundary $\psi_{-}$and $\psi_{+}$transform in completely different ways. In particular, in the limit $\varepsilon \rightarrow 0$ we recover for $\psi_{-}$the standard transformation rule for fermion with the Weyl weight $w$ under the conformal mappings:

$$
\delta \psi_{-}(\vec{x})=\xi^{i} \partial_{i} \psi_{-}+w\left(A_{k} x^{k}+D\right) \psi_{-}-\frac{1}{4} \eta^{k[i} \partial_{k} \xi^{j]} \gamma_{i j} \psi_{-},
$$

while $\psi_{+}(\vec{x})$ realizes a nonlocal representation of the conformal group. Since in CFT quasiprimary operators $\mathcal{O}(x)$ transform in local representations of the conformal group only $\psi_{-}$can be coupled on the boundary with a primary operator of the conformal weight $\Delta=\frac{d}{2}+m$. Thus, the transformation properties of $\psi_{ \pm}$imply that in the case $m>0$ we should treat $\psi_{-}$ and $\bar{\psi}_{+}$(analogously for $\bar{\psi}_{-}$and $\psi_{+}$) as the canonically conjugate coordinate and momentum respectively.

As was discussed in the Introduction in the Hamiltonian version of the AdS/CFT correspondence one should use an action of the form $\int(p \dot{q}-H(p, q))$. Action $S_{\psi}$ rewritten in such a form looks as

$$
\begin{aligned}
S_{\psi}(\alpha) & =\int_{M} d^{d+1} x \sqrt{-G}\left(-x_{0}\left(\bar{\psi}_{+} \partial_{0} \psi_{-}+\partial_{0} \bar{\psi}_{-} \psi_{+}\right)+\frac{\alpha}{2} \bar{\psi} \Gamma^{i} D_{i} \psi-\frac{\beta}{2} D_{i} \bar{\psi} \Gamma^{i} \psi-m \bar{\psi} \psi\right. \\
& \left.+\frac{d}{2}\left(\alpha \bar{\psi}_{-} \psi_{+}+\beta \bar{\psi}_{+} \psi_{-}\right)\right)+\frac{1}{2} \int_{M} d^{d+1} x \partial_{0}\left(\sqrt{-G} x_{0}\left(\alpha \bar{\psi}_{-} \psi_{+}+\beta \bar{\psi}_{+} \psi_{-}\right)\right)
\end{aligned}
$$


In passing to the Hamiltonian formulation the total derivative term in (3.40) should be omitted. Taking into account that

$$
\int_{M} d^{d+1} x \sqrt{-G}\left(\frac{\alpha}{2} \bar{\psi} \Gamma^{i} D_{i} \psi-\frac{\beta}{2} D_{i} \bar{\psi} \Gamma^{i} \psi\right)=\int_{M} d^{d+1} x \sqrt{-G}\left(\bar{\psi} \Gamma^{i} D_{i} \psi-\frac{\beta d}{2}\left(\bar{\psi}_{+} \psi_{-}-\bar{\psi}_{-} \psi_{+}\right)\right)
$$

we finally obtain the action that should be used in computing the Green functions:

$$
\mathbf{S}_{\psi}=\int_{M} d^{d+1} x \sqrt{-G}\left(-x_{0}\left(\bar{\psi}_{+} \partial_{0} \psi_{-}+\partial_{0} \bar{\psi}_{-} \psi_{+}\right)+\bar{\psi} \Gamma^{i} D_{i} \psi-m \bar{\psi} \psi+d \bar{\psi}_{-} \psi_{+}\right) .
$$

Note that this action does not depend on $\alpha$ and $\beta$ as one could expect from the very beginning.

In the Lagrangian picture the total derivative term can be compensated by adding to action (3.32) the following boundary term

$$
I_{\psi}=\frac{1}{2} \int_{\partial M} d^{d} x \sqrt{-\bar{g}}\left(\alpha \bar{\psi}_{-} \psi_{+}+\beta \bar{\psi}_{+} \psi_{-}\right) .
$$

For the free theory under consideration the on-shell value of the boundary term is equal to

$$
I_{\psi}=\frac{1}{2} \int_{\partial M} d^{d} x \sqrt{-\bar{g}} \bar{\psi} \psi
$$

It can be seen as follows. Since $\mathbf{S}_{\psi}$ does not depend on $\alpha$ one gets

$$
\mathbf{S}_{\psi}=S_{\psi}(\alpha)+\frac{1}{2} \int_{\partial M} d^{d} x \sqrt{-\bar{g}}\left(\alpha \bar{\psi}_{-} \psi_{+}+\beta \bar{\psi}_{+} \psi_{-}\right)=S_{\psi}(1)+\frac{1}{2} \int_{\partial M} d^{d} x \sqrt{-\bar{g}} \bar{\psi} \psi
$$

Now taking into account that on shell $S_{\psi}(\alpha)=0$ for any $\alpha$ one obtains the desired equality. The equality of (3.42) and (3.43) seems to hold in the case of an interacting theory as well.

In [5] (see also [10]) the boundary term (3.43) with arbitrary numerical coefficient was suggested as the necessary addition to the Dirac action (3.32) to produce the conformally invariant two-point function of the fermion fields in the boundary CFT. It comes from our analysis that the Hamiltonian version of the AdS/CFT correspondence not only reproduces this boundary term but predicts its numerical coefficient.

It remains to note that when $m<0$ the chiral components $\psi_{ \pm}$change places, i.e. $\psi_{+}$

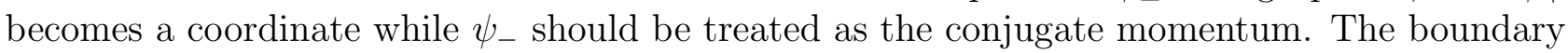
term will be given by the same expression (3.43) but with the opposite sign. In the case $m=0$ either $\psi_{+}$or $\psi_{-}$can be chosen as the coordinate and the sign of the boundary term depends only on this choice.

Thus, we have shown that using the Hamiltonian version of the AdS/CFT correspondence we can find all boundary terms necessary in the Lagrangian formulation. Our consideration can be easily extended to a supergravity model containing tensor fields in its spectrum. In particular, an interesting problem is to find all boundary terms for type $I I B$ supergravity on the $A d S_{5} \times S_{5}$ background. To this end one can use the covariant action found in [16, 17]. The solution of this problem would be the first step in establishing the conformal invariance of higher-point Green functions computed from the $A d S$ supergravity.

ACKNOWLEDGMENT The authors thank I.Y.Aref'eva and L.O.Chekhov for valuable discussions. We are also grateful to K.Sfetsos for drawing our attention to the revised version of [10] and for pointing out a misprint in the first version of our paper. This work has been supported in part by the RFBI grants N96-01-00608 and N96-01-00551. 


\section{References}

[1] J.Maldacena, "The large N limit of superconformal field theories and supergravity", hepth/9711200.

[2] G.G.Gubser,I.R.Klebanov and A.M.Polyakov, "Gauge theory correlators from noncritical string theory", hep-th/9802109.

[3] E.Witten, "Anti de Sitter space and holography", hep-th/9802150.

[4] I.Ya.Aref'eva and I.V.Volovich, On large N conformal field theories, field theories in anti-de Sitter space and singletons, hep-th/9803028.

[5] M.Henningson and K.Sfetsos, Spinors and the AdS/CFT correspondence, hep-th/9803251.

[6] W.Mück and K.S.Viswanathan, Conformal field theory correlators from classical scalar field theory on anti-de Sitter space, hep-th/9804035.

[7] D.Freedman, Samir D.Mathur, A.Matusis and L.Rastelli, Correlation functions in the $C F T_{d} / A d S_{d+1}$ correspondence, hep-th/9804058.

[8] H.Liu and A.A.Tseytlin, " $D=4$ Super Yang-Mills, $D=5$ gauged supergravity and $D=4$ conformal supergravity", hep-th/9804083.

[9] G.Chalmers, H.Nastase, K.Schalm and R.Siebelink, R-Current Correlators in $\mathcal{N}=4$ super YangMills theory from anti-de Sitter supergravity, hep-th/9805105.

[10] W.Mück and K.S.Viswanathan, Conformal field theory correlators from classical field theory on anti-de Sitter space II. Vector and Spinor fields, hep-th/9805145.

[11] A.Ghezelbash, K.Kaviani, S.Parvizi and A.Fatollahi, Interacting spinors - scalars and the ADS/CFT correspondence, hep-th/9805162.

[12] S.Lee, S.Minwalla, M.Rangamani, N.Seiberg, Three-point functions of chiral operators in $D=4$, $\mathcal{N}=4$ SYM at large $N$, hep-th/9806074.

[13] I.Ya.Aref'eva and I.V.Volovich, On the breaking of conformal symmetry in the AdS/CFT correspondence, hep-th/9804182.

[14] R.Kallosh and A. van Proeyen, Conformal symmetry of supergravities in AdS spaces, hepth/9804099.

[15] G.W.Gibbons and S.W.Hawking, "Action integrals and partition functions in quantum gravity", Phys.Rev. D15 (1977) 2752.

[16] G. Dall'Agata, K.Lechner and D.Sorokin, Covariant actions for the bosonic sector of $D=10 I I B$ supergravity, hep-th/9707044.

[17] G. Dall'Agata, K.Lechner and M.Tonin, $D=10, N=I I B$ supergravity: Lorentz-invariant actions and duality, hep-th/9806140. 